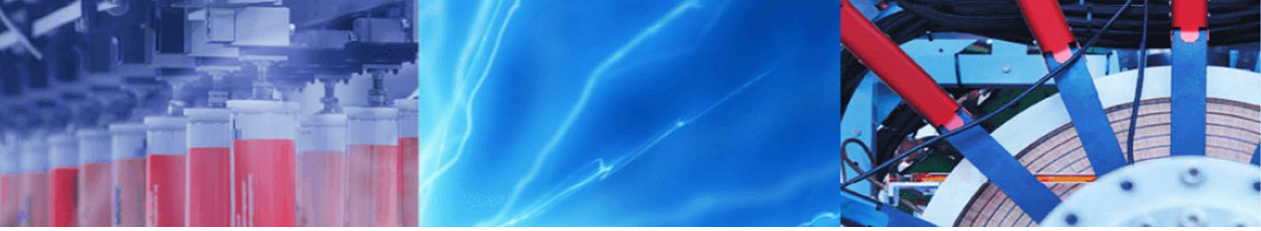

Research Article

\title{
Volatile organic compounds detection by electrical sensors using polyalkylthiophene-based Langmuir-Blodgett films
}

\author{
E. A. Silva ${ }^{1}$ M. L. Braunger ${ }^{1}$ A. Gregori ${ }^{2} \cdot$ C. A. Olivati $^{1}$
}

(c) Springer Nature Switzerland AG 2019

\begin{abstract}
Despite their environmental and health hazards, volatile organic compounds (VOCs) are present in our everyday life (e.g., fuels and solvents). Their detection is of paramount importance for environmental and occupational hazards monitoring. Among other technologies, semiconducting polymers show good capabilities in VOCs detection and identification, thanks to the variation of their electronic properties upon VOC exposure. We fabricated and characterized VOCs sensors depositing thin films of regioregular poly(3-alkylthiophene) derivatives (rr-P3ATs) and stearic acid (SA) onto gold interdigitated electrodes by Langmuir-Blodgett technique. Poly(3-butylthiophene) and poly(3-hexylthiophene) (P3HT) were mixed with SA at different ratios, and their electrical conductivity was used to optimize the film composition. We characterized the optoelectronic and morphological properties of these films, as well as their electrical response to dichloromethane (DCM), tetrahydrofuran and toluene VOC exposure. Both P3AT sensors showed distinct and characteristic responses, highlighting their ability to recognize different VOCs. Moreover, we investigated the sensors saturation and sensitivity to different VOCs. The sensors were still able to detect the VOCs after six cycles, with P3HT remarkably showing no saturation at all for DCM, and the characteristic single VOC response for subsequent exposure to the different VOCs.
\end{abstract}

Keywords Langmuir-Blodgett · Polyalkylthiophenes · Electrical conductivity · VOCs electrical sensor

\section{Introduction}

Volatile organic compounds (VOCs) are extensively utilized in fuels and as solvents [1], although they are hazardous indoor air pollutants, harmful to human health, whose chronic exposure causes assorted pulmonary illnesses [2, 3]. Moreover, some VOCs dispensation has been associated with ecological damage [4]. Hence, VOCs detection is essential for environmental and occupational hazards monitoring and safety application. In this area, conducting polymers can be used for VOCs detection and identification, and the polymer-based sensors are especially reliant on the variation of the polymer electrical conductivity under analyte vapor exposure [5].

Electroactive polymers with a large carrier delocalization-such as polypyrroles, polyaniline, polyacetylene and polythiophenes-assume outstanding significance due to their idiosyncratic electronic properties [6, 7]. Among these, regioregular polythiophenes possess air-stable conducting features, particularly appropriate for VOCs sensing [8]. The tunability of the side-chain groups on the polythiophene backbone gives access to a broad amount of appealing materials for VOCs sensing $[9,10]$. Additionally, small changes in conformational order and solid-state

Electronic supplementary material The online version of this article (https://doi.org/10.1007/s42452-019-0187-z) contains supplementary material, which is available to authorized users.

$\triangle$ C. A. Olivati, olivati@fct.unesp.br; E. A. Silva, eassuncaosilva@gmail.com; M. L. Braunger, malubraunger@yahoo.com.br; A. Gregori, alberto.gregori@iit.it | 'Departamento de Fisica, Faculdade de Ciências e Tecnologia, UNESP, Rua Roberto Simonsen, 305, Presidente Prudente 19060-900, Brazil. ${ }^{2}$ Center for Sustainable Future Technologies, Istituto Italiano di Tecnologia, via Livorno 60, 10144 Turin, Italy.

SN Applied Sciences (2019) 1:200 | https://doi.org/10.1007/s42452-019-0187-z 
organization result in a large range of conductivities, making them devices with exquisite sensitivity.

The Langmuir-Blodgett (LB) method allows to achieve and control a true molecular monolayer buildup [11], while saving materials compared to traditional fabrication methods [12]. In the LB technique, molecular or polymeric monolayers self-arrange on an aqueous surface, at the liquid-air interface, over compression and afterward transferred onto a substrate as coatings, with a thickness down to molecular dimensions [13]. LB film deposition is highly reproducible and gives the control over interparticle distance decisive to exploit the materials in various technological applications [14].

Polythiophenes, as other conjugated polymers, do not spread evenly over the water subphase in a Langmuir trough, due to their low-amphiphilic character, forming instead a rather rigid floating film, which hinders the deposition of the monolayers onto solid substrates [15, 16]. The introduction of amphiphilic molecules, such as fatty acids, effectively solves this issue. Acting as spacers, they stabilize the monolayers, which form less rigid films that can be easily transferred onto the substrate [17]. In a previous study, we reported on the same effect for different polyalkylthiophene derivatives, using polarization modulation infrared reflection-absorption spectroscopy [18]. Sakai et al. [19] showed the stabilizing effect of stearic acid on poly(p-phenylene vinylene) films.

In this paper, we analyze the ability of LB films fabricated from regioregular poly(3-alkylthiophene) derivatives and stearic acid to work as electrical sensors for three selected VOCs. Herein, we fabricated LB films of poly(3butylthiophene) and poly(3-hexylthiophene) mixed with stearic acid at different ratios. Their electrical properties were evaluated in order to optimize the composition that leads to the best electrical response. With this in mind, the selected films were then characterized optically and morphologically, and their electrical sensing capability toward dichloromethane, tetrahydrofuran and toluene was tested.

\section{Experimental}

\subsection{Materials}

Regioregular poly(3-butylthiophene) (P3BT) and poly(3hexylthiophene) (P3HT) and stearic acid (SA) used in this investigation were purchased from Sigma-Aldrich. Dichloromethane (DCM), tetrahydrofuran (THF) and toluene were purchased from Vetec. All chemicals were used as received, without further purification.

BK7 glass slides were exposed to hexamethyldisilazane (HMDS) vapor for $48 \mathrm{~h}$ in order to create a hydrophobic surface. Brazilian Nanotechnology National Laboratory
Table 1 Optimized LB deposition parameter for P3BT:SA films

\begin{tabular}{llll}
\hline SA percentage $(\mathrm{mol} \%)$ & 66 & 43 & 24 \\
Spread volume $(\mu \mathrm{L})$ & 250 & 250 & 500 \\
Compression rate $\left(\mathrm{mm} \mathrm{min}^{-1}\right)$ & 10 & 10 & 10 \\
Deposition surface pressure $\left(\mathrm{mN} \mathrm{m}^{-1}\right)$ & 30 & 35 & 40 \\
Speed upstroke $\left(\mathrm{mm} \mathrm{min}^{-1}\right)$ & 9 & 9 & 0.5 \\
Speed downstroke $\left(\mathrm{mm} \mathrm{min}^{-1}\right)$ & 8 & 9 & 0.2 \\
Average transfer ratio (up) & 0.9 & 0.7 & 0.7 \\
Average transfer ratio (down) & 0.7 & 0.6 & 0.2 \\
\hline
\end{tabular}

Table 2 Optimized LB deposition parameter for P3HT:SA films

\begin{tabular}{llll}
\hline SA percentage $(\mathrm{mol} \%)$ & 66 & 43 & 24 \\
Spread volume $(\mu \mathrm{L})$ & 250 & 250 & 250 \\
Compression rate $\left(\mathrm{mm} \mathrm{min}^{-1}\right)$ & 10 & 10 & 10 \\
Deposition surface pressure $\left(\mathrm{mN} \mathrm{m}^{-1}\right)$ & 30 & 30 & 40 \\
Speed up- and downstroke $\left(\mathrm{mm} \mathrm{min}^{-1}\right)$ & 11 & 11 & 0.3 \\
Average transfer ratio (up) & 0.8 & 0.9 & 0.5 \\
Average transfer ratio (down) & 0.7 & 0.9 & 0.4 \\
\hline
\end{tabular}

(LNNano) provided gold Interdigitated Electrode (IDE) on glass substrates.

\subsection{Optimization of LB films deposition}

Based on the work of Singhal et al. [20], we prepared an initial solution of P3AT:SA, 1:2 M in chloroform, with a total solid concentration of, respectively, 0.35 and $0.38 \mathrm{mg} \mathrm{m}^{-1}$ for the P3BT:SA and P3HT:SA. We then performed a systematic study, maintaining the same solid concentration, while varying the ratio between P3ATs and SA as 66,43 , 24 and 5 mol\%. LB films were fabricated by transferring P3AT:SA monolayers by vertical dipping in a pressure range of $30-40 \mathrm{mN} \mathrm{m}^{-1}$. SA is commonly used as additive $[15,21]$ to obtain good-quality films, without altering their optoelectronic properties.

The solutions were spread on the Langmuir through model KSV 5000, onto ultrapure water (Millipore system, resistivity $18.2 \mathrm{M} \Omega \mathrm{cm}$ ) forming Langmuir films. Before starting compression, a period of 15 min was granted to allow solvent evaporation. We recorded the $\pi-A$ isotherms $\left(22^{\circ} \mathrm{C}\right)$ of P3AT:SA at a barrier compression of $10 \mathrm{~mm} \mathrm{~min}^{-1}$. We transferred 25 monolayers forming LB films (vertical dipping) on both IDEs and hydrophobic BK7 slides for electrical, optical and morphological characterization. We report in Tables 1 and 2 the specific deposition parameters for P3BT:SA and P3HT:SA-LB films, respectively. We highlight the transfer ratio (TR), which is defined as the ratio between the decrease in monolayer area during a deposition stroke $\left(A_{1}\right)$ and the area of the substrate $\left(A_{s}\right)$; 
thus, $\mathrm{TR}=A_{l} / A_{\mathrm{S}}$ [22]. This parameter is crucial in evaluating the deposition success.

In order to investigate the effects of the film conformation on the electrical properties, we also deposited two films using the horizontal lifting, the so-called Langmuir-Schaefer (LS) films. One film was composed of P3AT only, which we could not fabricate via LB technique [23], and the other with $43 \mathrm{~mol} \% \mathrm{SA}$, which showed the highest conductivity in the whole study.

\subsection{Characterization}

We measured UV-Vis absorption spectra with a Varian Cary 50 spectrophotometer, in the range between 400 and $800 \mathrm{~nm}$. Surface morphology was characterized by contact mode atomic force microscopy (AFM) in air (Nanosurf Easyscan 2 Microscope). We then analyzed the topography and roughness of these $30 \times 30 \mu^{2}$ images through Gwyddion 2.34 software. Scanning electron microscopy (SEM) images were obtained on an EVO LS15 Carl Zeiss Microscope. Current versus voltage $(I-V)$ curves were obtained with a Source-Measure Unit Keithley 238, with a voltage ranging between 0 and $10 \mathrm{~V}$, steps of $1 \mathrm{~V}$ and delay between measured points of $100 \mathrm{~ms}$.

\subsection{Sensors}

We evaluated the electrical responses of P3AT LB films upon exposure to VOCs, using DC measurements $(I-t)$ at $5 \mathrm{~V}$ bias at room temperature. These VOCs were selected based on some characteristics, such as molecular structure, vapor pressure, dipole moment and relative permittivity [24]. We assessed the sensors response in a custommade test chamber in the presence of the VOCs in dynamic flow, dragged by nitrogen. A schematic representation of the custom-made setup is reported in Fig. 1. A constant flow at $60 \mathrm{NL} \mathrm{h}^{-1}$ (NL is a unit of mass for gases equal to the mass of $1 \mathrm{~L}$ at a pressure of $1 \mathrm{~atm}$ and at a standard temperature) was kept throughout the entire experiment. To measure the base level, we left the devices under nitrogen flow for $15 \mathrm{~min}$, followed by $15 \mathrm{~min}$ where the VOCs were dragged by the nitrogen flow, then returning to the same conditions before the VOCs presence. Thereby, the devices sensorial response was examined according to the current changes upon their exposure to the VOCs. Prior to data acquisition, the devices were left under pure nitrogen flow for $30 \mathrm{~min}$ in the chamber to achieve steady reading.

\section{Results and discussion}

\subsection{DC electrical measurements}

For an applied voltage $V$ on the devices, a reciprocal current I was obtained as response in these electrical measurements, and the $I-V$ curves are reported in Fig. 2. We were able to fit the data using a linear first-degree equation, and from the slope in the graph $(1 / R)$, it is possible to find the electrical resistance of each sensor $R$. We, then, calculated the conductivity $\sigma$ of the material using $R$ and the geometric relation for the interdigitated electrodes, the cell constant $k\left[\mathrm{~cm}^{-1}\right]$. The $k$ of a conductivity electrolyte sensor is defined as the proportionality factor between the specific resistance of the electrolyte and the measured resistance [25]. This constant is determined by the sensor geometry [16], and it is $5.1 \mathrm{~cm}^{-1}$ in this study [26]. Finally, the relation for conductivity is $\sigma=\frac{1}{R} \kappa$. We summarize the calculated conductivity values in Table 3 .

The conductivities of the LB films show the same trend with respect to the $S A$ amount, with maximum and minimum values observed for the 43 and $66 \mathrm{~mol} \%$ films, respectively. This behavior is due to the combined effect of two main factors: the insulating effect of the SA $[27,28]$ and the LB films quality (observed by the transfer

Fig. 1 Schematic representation of the customized VOCs detection system used in this investigation





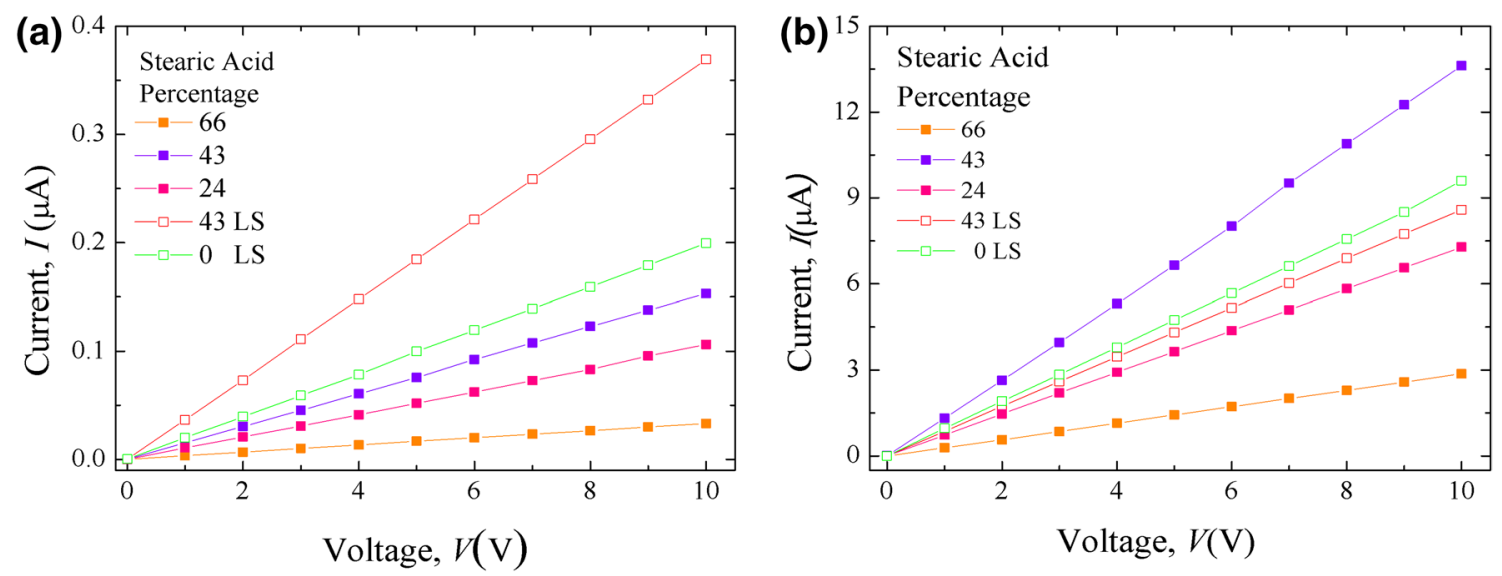

Fig. 2 Current versus voltage plots for LB and LS films of a P3BT and b P3HT, both in mixtures with stearic acid (SA) at different molar ratios

Table 3 Electrical conductivity for LB films of P3BT:SA and P3HT:SA at different molar ratios in interdigitated electrode devices

\begin{tabular}{lll}
\hline Stearic acid percentage & \multicolumn{2}{l}{ Conductivity $\sigma\left(\mathrm{S} \mathrm{m}^{-1}\right)$} \\
\cline { 2 - 3 } & P3BT & P3HT \\
\hline 66 & $1.69 \times 10^{-8}$ & $1.46 \times 10^{-6}$ \\
43 & $7.83 \times 10^{-8}$ & $6.97 \times 10^{-6}$ \\
24 & $5.39 \times 10^{-8}$ & $3.71 \times 10^{-6}$ \\
$0 \mathrm{LS}$ & $1.02 \times 10^{-7}$ & $4.85 \times 10^{-6}$ \\
$43 \mathrm{LS}$ & $1.88 \times 10^{-7}$ & $4.38 \times 10^{-6}$ \\
\hline
\end{tabular}

The conductivities for LS films are presented in italic

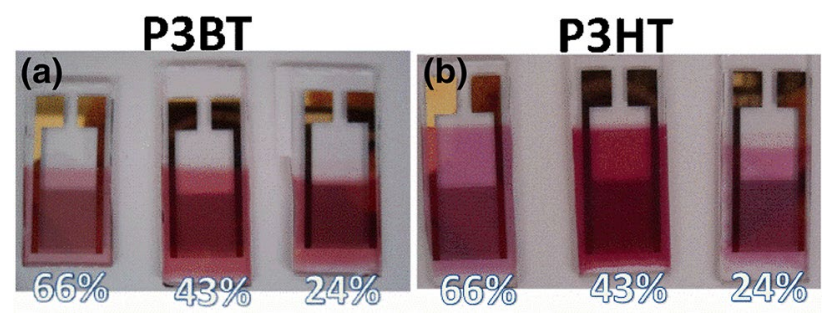

Fig. 3 LB films of a P3BT:SA and $\mathbf{b}$ P3HT:SA deposited onto gold IDE at different molar ratios

ratio of the different percentages as seen in Tables 1,2). Simply by eye inspection, the LB films containing the same number of layers (Fig. 3) clearly show how the SA content changes significantly the quality of the film. Therefore, the films containing $43 \mathrm{~mol} \%$ of SA presented an optimal point, once the amount of SA was enough to create a favorable assembling and its insulating behavior did not harm the conducting properties of the polymers. Chen et al. [29] also reported a study with P3HT/ insulating polymer blends and detected a maximum on conductivity for an optimal amount of P3HT. We also tried percentages in between, both $24-43 \mathrm{~mol} \%$ and 43-66 mol\%, but 43 mol\% gave the highest conductivity. Moreover, LS method was used to fabricate films with the higher conductivity ( $43 \mathrm{~mol} \% \mathrm{SA}$ ) and the pristine polymer in a way to verify whether the electrical outcome is due to the SA addition or film organization provided by the $L B$ technique.

P3HT-LS films present lower conductivity with respect to the corresponding P3HT-LB films, while the P3BT-LS and LB show the opposite trend. We attribute this behavior to the lower degree of organization of these latter P3BT-LB films, as observed during their deposition, and highlighted by the low TRs in Table 1 .

The conductivity values previously reported were acquired through different techniques, whereas P3BT revealed conductivities in an expanded range that goes from $10^{-7}$ to about $200 \mathrm{~S} \mathrm{~m}^{-1}$ [30-32], and among the reports for this polymer the results are from pristine, doped or in blends with other polymers or molecules. Now, for the P3HT the electric conductivities in the literature vary up to ten orders of magnitude from $10^{-7}$ to $10^{3} \mathrm{~S} \mathrm{~m}^{-1}$ considering the neutral, mixed and doped states [33-36]. Therefore, the conductivities acquired in this work are in the range within literature for the P3BT and P3HT films, which strongly depends on the measurement technique and electrode geometry $[32,34,35$, 37]. P3BT shows overall smaller conductivities than $\mathrm{P} 3 \mathrm{HT}$, regardless of the SA amount or deposition technique (LS or LB) used. This difference is in agreement with previous reports and is mainly attributed to how the side chain of such polymers affects the dimensionality and coplanarity of the conjugated main chain and therefore the interchain interaction $[30,31]$. 


\subsection{Optical and morphological characterization}

In Fig. 4, we compare the UV-Vis absorption spectra of P3BT:SA and P3HT:SA in chloroform solutions and in LB films. The solution spectra for both P3AT:SA mixtures are in agreement with reported results for the pristine polymers [38, 39], as expected after considering the stearic acid absorption spectra [40]. The films spectra maxima show a strong redshift of about 80 and $100 \mathrm{~nm}$ for P3BT:SA and $\mathrm{P} 3 \mathrm{HT}$ :SA, respectively. This shift is commonly attributed to the chains organization in the film [41], which is further confirmed by the shoulder at $590 \mathrm{~nm}$ [42]. This shoulder is clearly visible in the case of $\mathrm{P} 3 \mathrm{HT}$ and barely visible for P3BT. This reflects the different degree of crystallinity observed from the electrical characterization, confirming the distinct interchain interaction due to the polymer side chain.

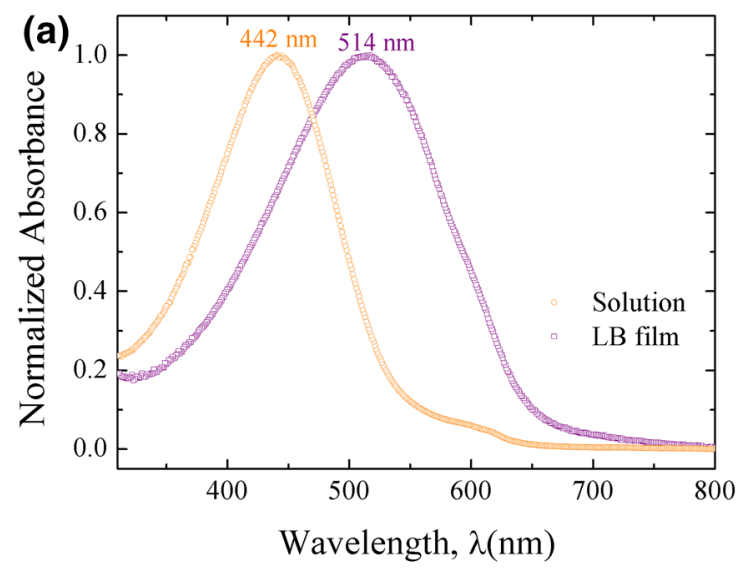

The AFM image of the IDE substrate revealed a rather homogeneous surface, with an average roughness value root mean square (RMS) of approximately $2.8 \mathrm{~nm}$. In Fig. 5 , we show the AFM and SEM images of LB and LS films of P3BT and P3HT with 43\% SA. We observed many large and widely spread clusters in both LB and LS films, which result in a high average roughness of $90-200 \mathrm{~nm}$. The P3BT:SA films show larger agglomerates and higher RMS values than P3HT:SA films. The P3HT- and P3BT-LB films possess similar average roughness $(\sim 155 \mathrm{~nm})$, while the P3HT-LS films have a reduced RMS of $90 \mathrm{~nm}$ opposite to the P3BTLS films with an RMS of $200 \mathrm{~nm}$. This is probably related to the distinct solubility of the two polymers in chloroform and to the films organization on the Langmuir trough coming from the specific interaction between each polymer with the SA. The aggregates and non-homogeneities also have a strong impact on the film conductivity observed

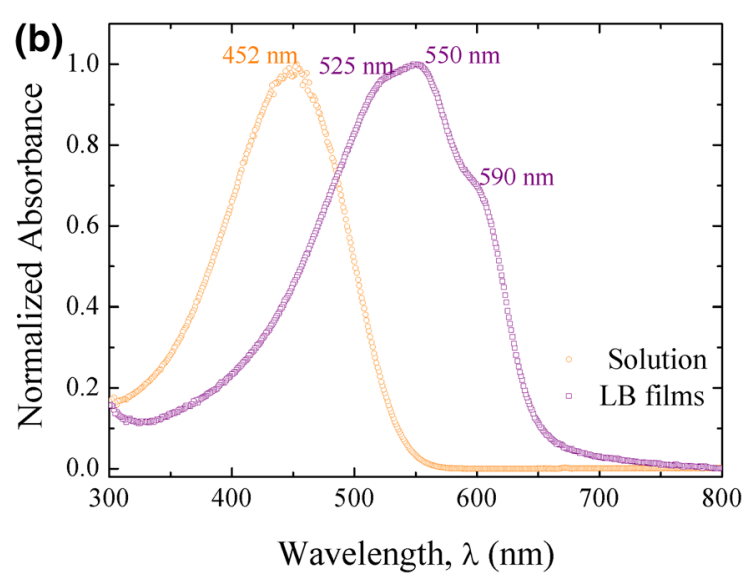

Fig. 4 Absorption spectra in the UV-visible range of chloroform solution and LB films of a P3BT:SA and b P3HT:SA, at a 43 mol\% of SA

(a) $0 \mu \mathrm{m} \quad 10 \quad 20$



(c) $0 \mu \mathrm{m} \quad 10 \quad 20$

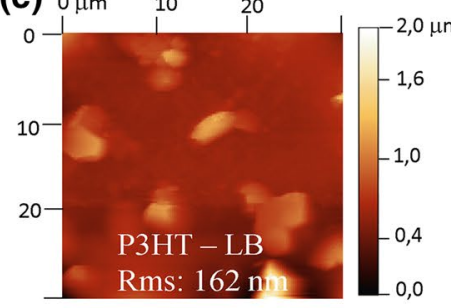

(b) $0 \mu \mathrm{m} \quad 10 \quad 20$
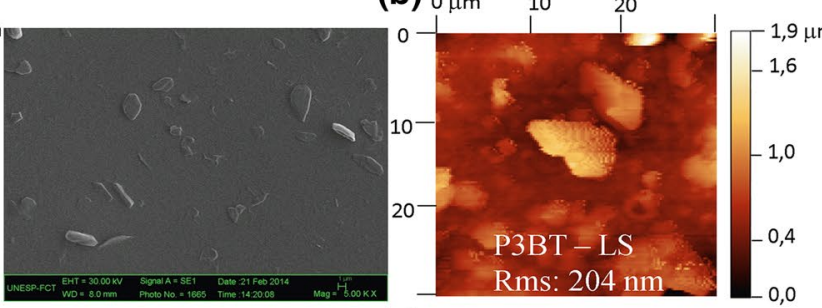

(d) $0 \mu \mathrm{m} \quad 10 \quad 20$

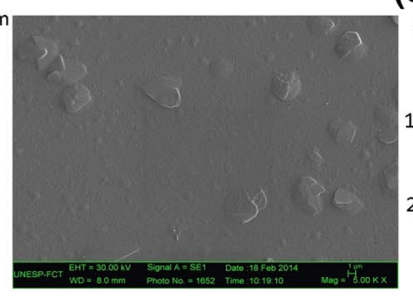



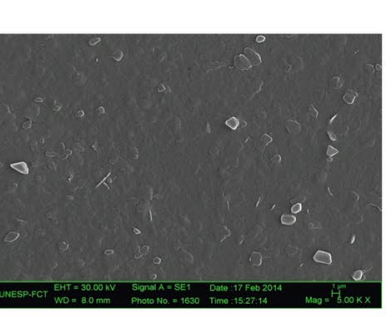

Fig. 5 AFM and SEM images for P3BT:SA a LB film and $\mathbf{b}$ LS film, and P3HT:SA c LB film and $\mathbf{d}$ LS film, containing 43 mol\% of SA 
on the electrical measurements, fact probably related to the change in conformation which can be noticed by the contrasting AFM images of the two P3AT:SA-LB films, since morphology impacts greatly the conductivity $[43,44]$.

\subsection{VOC sensors}

We obtained normalized current responses over time of the P3AT:SA sensors to DCM, THF and toluene. Normalization is used to analyze polymer sensor data so that sensors with different base resistances can be better compared. The normalization is based on Eq. (1) in which sensor response is given by:

$I_{\text {response }}=\frac{I_{\mathrm{s}}(t)-I_{\mathrm{s}}(0)}{I_{\mathrm{s}}(0)}$

where $I_{s}(t)$ is the sensor current during vapor exposure, and $I_{s}(0)$ is the initial current. Response sensitivities are measured as changes in the electric current of both polymers under controlled flow of a given VOC. Results are shown in Fig. 6.

The current variation was similar for both polymers, except in the case of DCM. Besides, only a partial recovery happens in all the cases, probably due to the chemisorption of the analytes into the films. We notice that the toluene response is the lowest among the tested VOCs, while the DCM reaches a high chemical response faster. The aforementioned effects may be explained by the rather larger toluene molar volume $\left(107.00 \mathrm{~cm}^{3} \mathrm{~mol}^{-1}\right)$ when compared to THF $\left(81.70 \mathrm{~cm}^{3} \mathrm{~mol}^{-1}\right)$ and DCM $\left(64.10 \mathrm{~cm}^{3} \mathrm{~mol}^{-1}\right)$. Such characteristics imply that the toluene penetration into the films is harder and slower, resulting in the lower electrical feedback, while the DCM diffuses easily within the films causing a rapid and intense response $[45,46]$.

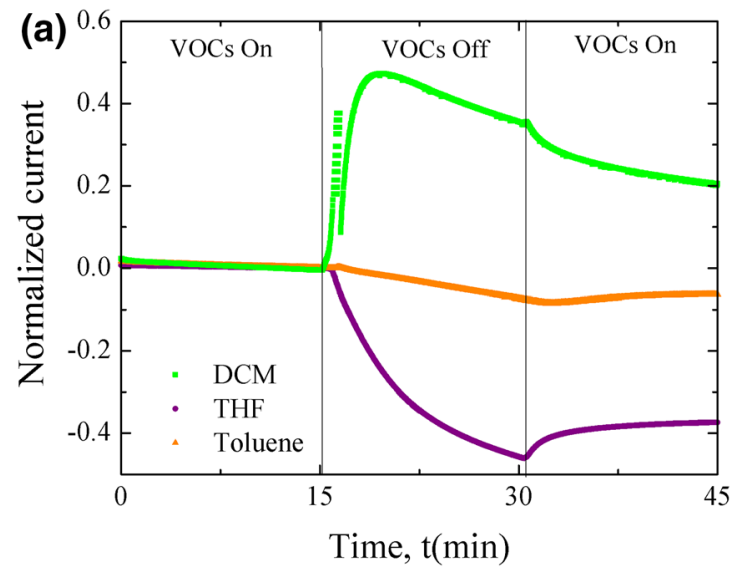

The polymer electrical response is directly dependent on the electric dipole moment of the vapors, as already observed in the literature [47-49]. The shapes of the graphs also revealed the selectivity property of the films to each VOC.

The sensing properties are highly reliant on the molecular interactions (bonding, chemical reactions, dipole interaction and van der Waals forces) between the analytes and the sensing materials, and on the film nanostructure. These characteristics are determined, among other things, by the polymer side chain [9]. The specific characteristics of films and analytes determine which of these mechanisms will contribute more significantly to the driving process, and they can be noticed by the different responses seen through the curves shapes and conductivity shift modulus.

The negative shifts seen for THF and toluene can be explained by the fact that they are both good solvents for P3ATs and dissolve the polymers, causing a swelling effect by the separation of the polymer molecules and film structures. As a result, a rise of the carrier hopping distance and barrier energy occurs, leading to the drop of conductivity as observed [47]. Moreover, the higher modulus current maximum found for THF may be related to the polar nature of this solvent, in contrast to the nonpolar structure of toluene. The DCM response is positive for P3BT and negative for P3HT. This quite distinct outcome of P3BT upon DCM exposure is due to its lower solubility compared to $\mathrm{P} 3 \mathrm{HT}$, as well as to its more closed structure in view of the shorter side chain length. Hence, the interaction of the butyl derivative with the analytes probably diminishes the polymer molecules distances, raising the intrachain (backbone) and interchain (hopping) carrier transport [9].

With the purpose to detect the films saturation, i.e., sensing capability after continued vapor exposure, a number of cycles were performed. The results can be seen in Fig. 7, where, in view of the similarities between the two

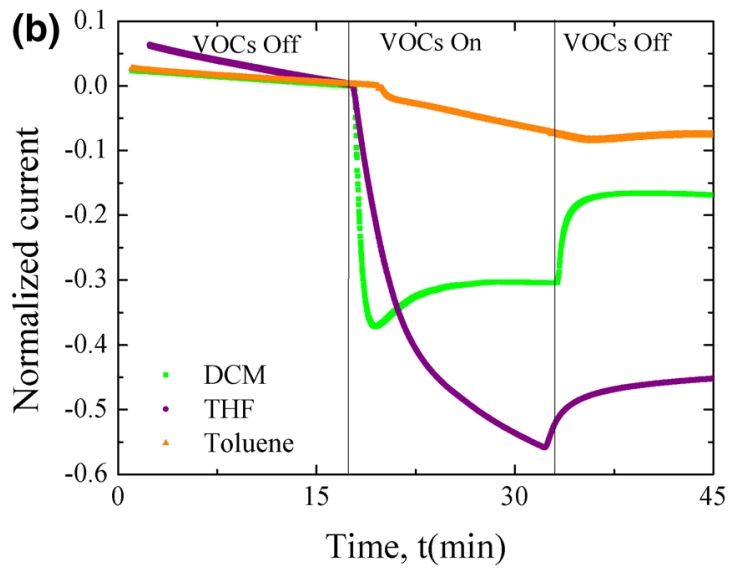

Fig. 6 Sensor responses for a P3BT:SA and $\mathbf{b}$ P3HT:SA to the VOC vapors of dichloromethane (DCM), tetrahydrofuran (THF) and toluene 


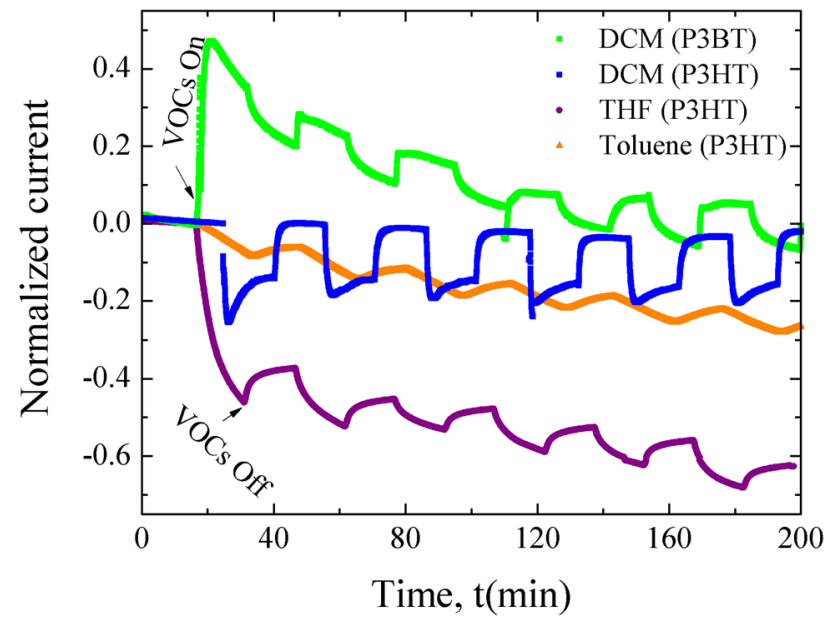

Fig. 7 Sensor responses to the VOC vapors of dichloromethane, tetrahydrofuran and toluene after 6 cycles

Table 4 Response times of the sensors to the VOCs for successive cycles

\begin{tabular}{lllll}
\hline Cycle & \multicolumn{4}{l}{ Response time (s) } \\
\cline { 2 - 5 } & DCM (P3BT) & DCM (P3HT) & THF & Toluene \\
\hline 1 & 166 & 60 & 643 & 798 \\
2 & 22 & 74 & 710 & 847 \\
3 & 27 & 61 & 703 & 822 \\
4 & 181 & 28 & 723 & 810 \\
5 & 285 & 67 & 648 & 890 \\
6 & 162 & 71 & 632 & 846 \\
\hline
\end{tabular}

polymers response, for the sake of clarity, merely the $\mathrm{P} 3 \mathrm{HT}$ curves for THF and toluene are shown, while DCM detection for both polymers. Complete results are reported in SI (Fig. S1).

Concerning the THF and toluene signals, we observe that despite the incomplete recovery, the P3AT-based devices keep detecting the VOCs presence. The DCM response of $\mathrm{P} 3 \mathrm{BT}$ shows neither the previously observed saturation nor a complete recovery. However, it tends to stabilize over time. On the contrary, the P3HT feedback to this vapor is rather reversible. Table 4 depicts the response time for each cycle, defined as the time required for the current to reach $90 \%$ of its maximum value after the gas was introduced in the test chamber. From the table, we can see that DCM shows the fastest response times, most probably arising from the smaller molar volume that facilitates its diffusion within the films. Still, the two polymers show a different behavior upon DCM exposure. P3BT has a first cycle with $166 \mathrm{~s}$ of response time, which decreases in the next two cycles, although it seems a temporary effect of the molecules diffusion until the sensor reaches the stabilization. For P3HT, with exception of the fourth cycle, the response times to DCM vapor were consistent, demonstrating the sensing reliability of $\mathrm{P} 3 \mathrm{HT}$ to this VOC. Since there is no plateau in the due time for THF and toluene, the response times are quite high. However, THF maximum current is roughly four times higher than the correspondent toluene value. Additionally, toluene hindered diffusion into the film, due to its nonpolar characteristic and high molar volume, caused the longer response times and reduced maximum modulus of detection.

We also investigated their ability to identify a VOC after a different one has passed, and they exhibited high selectivity nature by replicating the responses displayed for the separate cases. These results can be observed in SI (Fig. S2).

\section{Conclusions}

Electrical sensors consisting in LB films of regioregular polyalkylthiophenes mixed with stearic acid onto gold interdigitated electrodes responded to different VOC vapors. By the electrical, optical and morphological characterization, we could observe that the stearic acid did not affect the optoelectronic properties of the polymers, while improving their deposition via LB method. The polythiophenes sensing responses showed distinct patterns and maxima conductivities, demonstrating the sensor capability to differentiate between the VOCs. We described the possible mechanisms and the interactions between the polymers and the vapors responsible for the changes in electrical properties. Dichloromethane vapor had a peculiar impact over the polyalkylthiophene devices, in contrast to the other VOCs. Finally, in a proof-of-concept experiment, P3BT and P3HT LB thin films, with well-organized structure, showed high potential to be applied as VOCs sensing devices.

Acknowledgements The authors are grateful for the financial support of the Brazilian agencies CAPES, FAPESP [Grant Number 2010/200941], INEO/CNPq and LNNano (CNPEM) for providing gold IDEs [Project LMF 16557].

\section{Compliance with ethical standards}

Conflict of interest The authors declare that they have no conflict of interest.

\section{References}

1. Wang B, Sonar P, Manzhos S, Haick H (2017) Diketopyrrolopyrrole copolymers based chemical sensors for the detection and discrimination of volatile organic compounds. Sens Actuators B Chem 251:49-56. https://doi.org/10.1016/j.snb.2017.04.167 
2. Dolai S, Bhunia SK, Beglaryan SS et al (2017) Colorimetric polydiacetylene-aerogel detector for volatile organic compounds (VOCs). ACS Appl Mater Interfaces 9:2891-2898. https://doi. org/10.1021/acsami.6b14469

3. Spinelle L, Gerboles M, Kok G et al (2017) Review of portable and low-cost sensors for the ambient air monitoring of benzene and other volatile organic compounds. Sensors. https:// doi.org/10.3390/s17071520

4. Dolai S, Bhunia SK, Jelinek R (2017) Carbon-dot-aerogel sensor for aromatic volatile organic compounds. Sens Actuators B Chem 241:607-613. https://doi.org/10.1016/j.snb.2016.10.124

5. Persaud K (2005) Polymers for chemical sensing. Mater Today 8:38-44. https://doi.org/10.1016/S1369-7021(05)00793-5

6. Rella R, Siciliano P, Quaranta F et al (2000) Gas sensing measurements and analysis of the optical properties of poly[3-(butylthio) thiophene] Langmuir-Blodgett films. Sens Actuators B Chem 68:203-209. https://doi.org/10.1016/S0925-4005(00)00430-5

7. Baimpos T, Boutikos P, Nikolakis V, Kouzoudis D (2010) Physical A polymer-Metglas sensor used to detect volatile organic compounds. Sens Actuators A Phys 158:249-253. https://doi. org/10.1016/j.sna.2010.01.020

8. Kumar V, Kim K-H, Kumar P et al (2017) Functional hybrid nanostructure materials: advanced strategies for sensing applications toward volatile organic compounds. Coord Chem Rev 342:80105. https://doi.org/10.1016/j.ccr.2017.04.006

9. Li B, Santhanam S, Schultz L et al (2007) Inkjet printed chemical sensor array based on polythiophene conductive polymers. Sens Actuators B Chem 123:651-660. https://doi.org/10.1016/j. snb.2006.09.064

10. Zhao X (2005) Synthesis, characterization and structure dependence of thermochromism of polythiophene derivatives. J Mater Sci 40:3423-3428

11. Lo CK, Wang CY, Oosterhout SD et al (2018) Langmuir-Blodgett thin films of diketopyrrolopyrrole-based amphiphiles. ACS Appl Mater Interfaces 10:11995-12004. https://doi.org/10.1021/ acsami.7b18239

12. Reculusa S, Ravaine S (2003) Synthesis of colloidal crystals of controllable thickness through the Langmuir-Blodgett technique. Chem Mater 15:598-605. https://doi.org/10.1021/cm021 $242 \mathrm{w}$

13. Kim MS, Ma L, Choudhury $S$ et al (2016) Fabricating multifunctional nanoparticle membranes by a fast layer-by-layer Langmuir-Blodgett process: application in lithium-sulfur batteries. J Mater Chem A 4:14709-14719. https://doi.org/10.1039/c6ta0 $6018 \mathrm{~h}$

14. Martín-García B, Velázquez MM (2014) Nanoparticle self-assembly assisted by polymers: the role of shear stress in the nanoparticle arrangement of Langmuir and Langmuir-Blodgett films. Langmuir 30:509-516. https://doi.org/10.1021/la404834b

15. Niu JZ, Cheng G, Li Z et al (2008) Poly (3-dodecylthiophene) Langmuir-Blodgett films: preparation and characterization. Colloids Surfaces A Physicochem Eng Asp 330:62-66. https://doi. org/10.1016/j.colsurfa.2008.07.041

16. Braunger ML, da Silva EA, Awada $\mathrm{H}$ et al (2018) Langmuir and Langmuir-Blodgett films of low-bandgap polymers. Polym Int 67:1028-1034. https://doi.org/10.1002/pi.5604

17. Hill J, Heriot SY, Worsfold O et al (2004) Controlled Förster energy transfer in emissive polymer Langmuir-Blodgett structures. Phys Rev B 69:041303. https://doi.org/10.1103/PhysR evB.69.041303

18. da Silva EA, Caseli L, de Olivati AC (2017) Organization of polythiophenes at ultrathin films mixed with stearic acid investigated with polarization-modulation infrared reflection-absorption spectroscopy. Colloids Surfaces A Physicochem Eng Asp 529:628-633. https://doi.org/10.1016/j.colsurfa.2017.06.035
19. Sakai A, Wang SH, Péres LO, Caseli L (2011) Controlling the luminescence properties of poly( $p$-phenylene vinylene) entrapped in Langmuir and Langmuir-Blodgett films of stearic acid. Synth Met 161:1753-1759. https://doi.org/10.1016/j.synth met.2011.06.019

20. Singhal R, Chaubey A, Srikhirin T et al (2003) Immobilization of glucose oxidase onto Langmuir-Blodgett films of poly-3-hexylthiophene. Curr Appl Phys 3:275-279. https://doi.org/10.1016/ S1567-1739(02)00215-8

21. Sze H, Chan ON, Ng SIUC (1998) Synthesis, characterization and applications of thiophene-based functional polymers. Prog Polym Sci 23:1167-1231

22. Roberts GG (1990) Potential applications of Langmuir-Blodgett films. In: Roberts G (ed) Langmuir-Blodgett films. Springer, Boston, MA. https://doi.org/10.1007/978-1-4899-3716-2_7

23. Sharma SK, Singhal R, Malhotra BD et al (2004) Biosensor based on Langmuir-Blodgett films of poly(3-hexyl thiophene) for detection of galactose in human blood. Biotechnol Lett 26:645647. https://doi.org/10.1023/B:BILE.0000023023.19651.01

24. Sigma Aldrich Solvent Center. https://www.sigmaaldrich.com/ chemistry/solvents.html. Accessed 6 Feb 2018

25. Olthuis W, Streekstra W, Bergveld P (1995) Theoretical and experimental determination of cell constants of planar-interdigitated electrolyte conductivity sensors. Sens Actuators B Chem 24:252-256. https://doi.org/10.1016/0925-4005(95)85053-8

26. Volpati $D$, MacHado AD, Olivati CA et al (2011) Physical vapor deposited thin films of lignins extracted from sugar cane bagasse: morphology, electrical properties, and sensing applications. Biomacromolecules 12:3223-3231. https://doi. org/10.1021/bm200704m

27. Bertrand Y, Hoang LC (2004) Vegetable oils as substitute for mineral insulating oils in medium-voltage equipments. Sess CIGRE 1-6

28. Spohner M (2012) A Study of the properties of electrical insulation oils and of the components of natural oils. Acta Polytech 52:100-105

29. Chen J, Chen Z, Qu Y et al (2015) Large interfacial area enhances electrical conductivity of poly(3-hexylthiophene)/insulating polymer blends. RSC Adv 5:1777-1784. https://doi.org/10.1039/ C4RA12804D

30. Xu Y, Liu Y, Wu J, Zhu D (1998) Preparation and electrical conductivity of Langmuir-Blodgett films of poly(3-alkylthiophene) s. J Appl Polym Sci 69:1-6. https://doi.org/10.1002/(SICI)10974628(19980705)69:1\%3c1:AID-APP1\%3e3.0.CO;2-N

31. Sentein C, Mouanda B, Rosilio A, Rosilio C (1996) Influence of stereoregularity on the photoinitiated electrical conductivity of poly(3-alkylthiophenes). Synth Met 83:27-37. https://doi. org/10.1016/S0379-6779(97)80049-5

32. Chen G, Richardson TJ (1996) Overcharge protection for rechargeable lithium polymer electrolyte batteries. J Electrochem Soc 143:3992. https://doi.org/10.1149/1.1837326

33. Obrzut J, Page KA (2009) Electrical conductivity and relaxation in poly(3-hexylthiophene). Phys Rev B Condens Matter Mater Phys 80:1-7. https://doi.org/10.1103/PhysRevB.80.195211

34. Duong DT, Wang C, Antono E et al (2013) The chemical and structural origin of efficient p-type doping in $\mathrm{P} 3 \mathrm{HT}$. Organ Electron 14:1330-1336. https://doi.org/10.1016/j.orgel.2013.02.028

35. Panzer MJ, Frisbie CD (2006) High carrier density and metallic conductivity in poly(3-hexylthiophene) achieved by electrostatic charge injection. Adv Funct Mater 16:1051-1056. https:// doi.org/10.1002/adfm.200600111

36. Braunger $\mathrm{ML}$, Alessio $\mathrm{P}$, Furini $\mathrm{LN}$ et al (2017) Influence of the supramolecular arrangement in the electrical conductivity of poly(thiophene) thin films. J Nanosci Nanotechnol 17:460-466. https://doi.org/10.1166/jnn.2017.12667 
37. Singh RK, Kumar J, Singh R et al (2007) Micromorphology, photophysical and electrical properties of pristine and ferric chloride doped poly(3-hexylthiophene) films. Mater Chem Phys 104:390396. https://doi.org/10.1016/j.matchemphys.2007.01.024

38. Chen M, Li M, Wang $\mathrm{H}$ et al (2013) Side-chain substitution of poly(3-hexylthiophene) (P3HT) by PCBM via postpolymerization: an intramolecular hybrid of donor and acceptor. Polym Chem 4:550. https://doi.org/10.1039/c2py20651j

39. Ma J, Geng Y, Hashimoto K, Tajima K (2013) Synthesis and characterization of end-functionalized poly(3-butylthiophene) with semifluoroalkyl chains. Macromol Chem Phys 214:1326-1331. https://doi.org/10.1002/macp.201300081

40. Gomez NA, Abonia R, Cadavid H, Vargas IH (2011) Chemical and spectroscopic characterization of a vegetable oil used as dielectric coolant in distribution transformers. J Braz Chem Soc 22:2292-2303. https://doi.org/10.1590/S0103-5053201100 1200009

41. Dicker G, Savenije TJ, Huisman B-H et al (2003) Photoconductivity enhancement of poly(3-hexylthiophene) by increasing inter- and intra-chain order. Synth Met 137:863-864. https:// doi.org/10.1016/S0379-6779(02)01092-5

42. Trznadel M, Pron A, Zagorska M et al (1998) Effect of molecular weight on spectroscopic and spectroelectrochemical properties of regioregular poly(3-hexylthiophene). Macromolecules 31:5051-5058. https://doi.org/10.1021/ma970627a

43. Inceoglu S, Rojas AA, Devaux D et al (2014) Morphology-conductivity relationship of single-ion-conducting block copolymer electrolytes for lithium batteries. ACS Macro Lett 3:510-514. https://doi.org/10.1021/mz5001948
44. Jönsson SKM, Birgerson J, Crispin X et al (2003) The effects of solvents on the morphology and sheet resistance in poly $(3,4-$ ethylenedioxythiophene)-polystyrenesulfonic acid (PEDOTPSS) films. Synth Met 139:1-10. https://doi.org/10.1016/S0379 $-6779(02) 01259-6$

45. Acikbas Y, Capan R, Erdogan M et al (2017) Optical characterization and swelling behaviour of Langmuir-Blodgett thin films of a novel poly[(styrene (ST)-co-glycidyl methacrylate (GMA)]. Sens Actuators B Chem 241:1111-1120. https://doi.org/10.1016/j. snb.2016.10.025

46. Acikbas Y, Taktak F, Capan R et al (2018) An optical vapor sensor based on amphiphilic block copolymer Langmuir-Blodgett films. IEEE Sens J 18:5313-5320. https://doi.org/10.1109/ JSEN.2018.2833491

47. Li B, Sauvé G, lovu MC et al (2006) Volatile organic compound detection using nanostructured copolymers. Nano Lett 6:15981602. https://doi.org/10.1021/nl060498o

48. Shokuhi Rad A (2015) Application of polythiophene to methanol vapor detection: an ab initio study. J Mol Model 21:2-7. https:// doi.org/10.1007/s00894-015-2832-9

49. Janzen MC, Ponder JB, Bailey DP et al (2006) Colorimetric sensor arrays for volatile organic compounds. Anal Chem 78:35913600. https://doi.org/10.1021/ac052111s

Publisher's Note Springer Nature remains neutral with regard to jurisdictional claims in published maps and institutional affiliations. 